\title{
Blood lead level modifies the association between dietary antioxidants and oxidative stress in an urban adult population
}

\author{
Yun-Chul Hong ${ }^{1}$, Se-Young $\mathrm{Oh}^{2 *}$, Sung-Ok Kwon ${ }^{2}$, Min-Seon Park ${ }^{3}$, Ho Kim ${ }^{4}$, Jong-Han Leem ${ }^{5}$ \\ and Eun-Hee $\mathrm{Ha}^{6}$ \\ ${ }^{1}$ Department of Preventive Medicine, Seoul National University College of Medicine, Institute of Environmental Medicine, \\ Seoul National University Medical Research Center, Seoul, Republic of Korea \\ ${ }^{2}$ Department of Food and Nutrition, Research Institute of Human Ecology, Kyung Hee University, Seoul, Republic of Korea \\ ${ }^{3}$ Department of Family Medicine, Seoul National University Hospital, Seoul, Republic of Korea \\ ${ }^{4}$ Department of Epidemiology and Biostatistics, School of Public Health, Seoul National University, Seoul, Republic of Korea \\ ${ }^{5}$ Department of Occupational and Environmental Medicine, Inha University Hospital, Incheon, Republic of Korea \\ ${ }^{6}$ Department of Preventive Medicine, Ewha Womans University, Seoul, Republic of Korea
}

(Submitted 1 September 2011 - Final revision received 30 January 2012 - Accepted 1 February 2012 - First published online 2 April 2012)

\begin{abstract}
Oxidative stress may be affected by lead exposure as well as antioxidants, yet little is known about the interaction between dietary antioxidants and blood lead levels (BLL) on oxidative stress level. We investigated the interaction between dietary antioxidants and BLL on oxidative stress level. As part of the Biomarker Monitoring for Environmental Health conducted in Seoul and Incheon, Korea, between April and December 2005, we analysed data from 683 adults (female $=47 \cdot 4 \%$, mean age 51.4 (sD 8.4) years) who had complete measures on BLL, dietary intakes and oxidative stress marker (urinary 8-hydroxy-2'-deoxyguanosine (8-OHdG)). Dietary intakes were assessed by a validated semi-quantitative FFQ, BLL was measured using atomic absorption spectrophotometry, and 8-OHdG by ELISA. Multivariate linear regression analyses were used to evaluate the influence of BLL on the association between dietary antioxidants and 8-OHdG. Geometric means of BLL and 8-OHdG concentrations were 4.1 (SD 1.5) $\mu \mathrm{g} / \mathrm{dl}$ and 5.4 (SD 1.9) $\mu \mathrm{g} / \mathrm{g}$ creatinine, respectively. Increases of vitamins C and E were significantly associated with the decrease of $\log 10$ 8-OHdG in the adults from the lowest quartile of the BLL group ( $\leq 3 \cdot 18 \mu \mathrm{g} / \mathrm{dl}$, geometric mean $=2.36 \mu \mathrm{g} / \mathrm{dl})$ than those of the highest quartile BLL group $(>5.36 \mu \mathrm{g} / \mathrm{dl}$, geometric mean $=6.78 \mu \mathrm{g} / \mathrm{dl})$. Regarding antioxidant-related foods, vegetables excluding kimchi showed a higher inverse relationship with 8-OHdG in the lowest quartile BLL group than the highest group. These findings suggest a rationale for lowering the BLL and increasing the intake of dietary antioxidants in the urban population in Korea.
\end{abstract}

Key words: Diet: Antioxidants: Blood lead levels: Oxidative stress: Urban populations

Chronic exposure to environmental chemicals, adversely affecting the health of large numbers of people, is a global problem. Among the chemicals, lead continues to pose adverse health effects because it is related to a broad range of physiological, biochemical and behavioural dysfunctions ${ }^{(1)}$. Lead induces oxidative stress to the brain, heart, kidney and reproductive organs by affecting membranes, DNA and antioxidant defence systems of cells ${ }^{(1)}$. Aside from lipid peroxidation reactions evidenced by elevations in malondialdehyde, lead appears to enhance oxidative damage to DNA as shown by an increase of 8-hydroxydeoxyguanosine $(8-\mathrm{OHdG})^{(2-4)}$. In epidemiological studies, blood lead levels (BLL) have been reported to have adverse health impacts such as oxidative stress, mortality and raised homocysteine levels ${ }^{(5-7)}$.

Oxidative stress is implicated in the aetiology of many diseases and considered as an important pathophysiological process ${ }^{(8,9)}$. Antioxidant nutrients are thought to modulate oxidative damage by influencing a balance between free radical production and antioxidant capacity ${ }^{(8)}$. A number of studies have shown that antioxidant nutrients such as vitamin $\mathrm{C}$, vitamin $\mathrm{E}$ and/or $\beta$-carotene may decrease the biomarkers of oxidative stress, although some studies indicate a harmful effect of high-dose antioxidant supplements, particularly $\beta$-carotene ${ }^{(8,10)}$. The inverse association between vitamin $\mathrm{C}$ intake and lead exposure has also been reported ${ }^{(11)}$.

Abbreviations: 8-OHdG, 8-hydroxy-2'-deoxyguanosine; BLL, blood lead level.

*Corresponding author: S.-Y. Oh, fax +82 2959 0649, email seyoung@khu.ac.kr 
A few studies have examined the interaction between diet and environmental exposure with regard to health. Arora et $a{ }^{(12)}$ found maternal dietary PUFA as a modifier in the transfer of lead from bone to breast milk. A possible modifying effect of arsenic exposure on the relationship between nutrition and arsenic-induced health outcomes has also been suggested $^{(13)}$. Even though there were a few studies examining the interaction between nutrition and environmental exposure on health outcomes, the relationship between dietary antioxidants and oxidative stress has not been investigated in terms of BLL. The Biomarker Monitoring for Environmental Health was conducted to examine the health effects of environmental chemical exposure in the adult population. As part of the investigation, we evaluated whether the association between dietary antioxidants and oxidative stress in urban adults is modified by the degree of lead exposure.

\section{Materials and methods}

\section{Participants and data collection}

The present study was conducted according to the guidelines laid down in the Declaration of Helsinki and all procedures involving human subjects were approved by the Institutional Review Board of the Ewha Womans University Hospital and Inha University Hospital. Written informed consent was obtained from all participants when they visited the hospitals for health check-ups. Briefly, between April and December 2005, the Biomarker Monitoring for Environmental Health (principal investigator, Y.-C. H.) recruited 1131 adults, 40 years old or more, from two large cities, Seoul and Incheon, in Korea, who agreed to participate. Because we did not limit to one person per family, married couples could enrol into the study. We chose the cities because they are large cities with population sizes over 2.5 million and close enough to share the same urban lifestyle. Seoul is much larger and more metropolitan than Incheon, which is a harbour city on the west coast of the Korean Peninsula.

Of those 1131 participants, we excluded 124 adults with a previous history of disease that could affect the levels of oxidative stress markers, such as cancer ( $n$ 31), stroke ( $n$ 14), myocardial infarction ( $n 32)$, current medication for tuberculosis ( $n$ 3), acute hepatitis ( $n$ 2), chronic bronchitis $(n 9)$ and arthritis ( $n$ 33). We further excluded 292 adults whose BLL were missing, and thirty-two adults whose energy intake was either less than $2092 \mathrm{~kJ}$ ( $500 \mathrm{kcal})$ or greater than $20920 \mathrm{~kJ}(5000 \mathrm{kcal})^{(14)}$. As a result, a total of 683 adults were included in the data analyses. There was no difference of BLL and dietary intakes between our participants and the 124 adults excluded from the analyses due to their health conditions. The remaining 324 adults excluded due to lack of BLL or inappropriate energy intake values had lower proportions of males, smokers and drinkers and received less education than those included in the analyses.

Trained interviewers collected information on demographics, lifestyle habits, medical history, dietary intake and environmental exposure using a structured questionnaire. The height and weight of each subject were assessed according to standard recommended procedures. After the interviews, fasting blood and single urine samples were collected and stored at $-70^{\circ} \mathrm{C}$ until analysis. A sample of up to $3 \mathrm{ml}$ of each participant's blood was collected in a BD vacutainer (Becton Dickinson Company) containing K2 EDTA (Becton Dickinson Company) and preserved at $-20^{\circ} \mathrm{C}$.

\section{Measurement of biomarkers}

As an oxidative stress biomarker, urinary levels of $8-\mathrm{OHdG}$ were measured using the New 8-OHdG Check ELISA kit (JaICA) according to the manufacturer's instructions. The levels were divided by urinary creatinine concentrations to adjust the dilution in urine.

We used whole blood for the measurement of blood lead concentrations. Blood samples were diluted with $0 \cdot 1 \%$ Triton X-100 solution, and then analysed by atomic absorption spectrometry on an Analyst 100 instrument (Perkin-Elmer) using a graphite tube atomiser (HGA 800; Perkin-Elmer $\mathrm{GmbH})$. Lead concentrations were quantified in ${ }^{2} \mathrm{H}$ background correction mode. The limit of detection was $0.097 \mu \mathrm{g} / \mathrm{dl}$. The respective intra- and inter-assay CV for the blood lead concentrations were 2.9 and $5.3 \%$ and those for the urinary 8 -OHdG concentrations were $5 \cdot 2$ and $7 \cdot 5 \%$.

We measured urinary creatinine levels using a HITACHI 7600 instrument (HITACHI). Briefly, $10 \mathrm{ml}$ of urine and $300 \mathrm{ml}$ of picric acid solution (Wako) were mixed at room temperature for $3 \mathrm{~min}$, and absorbance of the solution was measured at $505 \mathrm{~nm}$. Following this, $75 \mu \mathrm{l}$ of alkaline solution (Wako) were added for $4 \mathrm{~min}$, and absorbance of the solution was measured at $570 \mathrm{~nm}$. The mean of the two values measured at 505 and $570 \mathrm{~nm}$ was used as the creatinine level.

\section{Dietary assessment}

We assessed usual dietary intake by a validated semi-quantitative FFQ used in the Korean Genome Epidemiologic Study ${ }^{(15)}$ Respective median correlation coefficients were 0.45 and $0.39(P<0.01)$ for reproducibility and validity of this instrument ${ }^{(15)}$. The FFQ contains 103 food items with nine nonoverlapping frequency response categories, ranging from 'rarely use' to have 'three or more times per day' in reference to the preceding year. For fruit items, participants were asked to mark one category among four on how long they ate the items: 3, 6, 9 and 12 months. For all foods, the possibility of specifying the use of a small, average or large portion was offered. For easy understanding of portion size, we provided pictures on serving size for food items on their own pages.

The amount of each food item included in the FFQ was converted into grams, from which the daily intakes of nutrients were calculated using DS 24 (Human Nutrition Lab, Seoul National University \& AI/DB Lab, Sookmyung Women's University, 1996). For fruit and vegetable intakes, daily servings were calculated based on intake frequencies and portion size options. We divided vegetable intake into kimchi and other vegetables due to the relatively high $\mathrm{Na}$ content of kimchi. 


\section{Potential confounders}

We assessed smoking (never, past smoker, current smoker), alcohol consumption (never, past drinker, current drinker), nutrition supplement intake (yes/no), exercise (yes/no) and educational levels (nine categories from no schooling to graduate school or more) with a structured questionnaire. BMI was calculated using height and weight values.

\section{Statistical analysis}

BLL and 8-OHdG values were log10-transformed because of their skewed distribution. Intake variables were log-transformed due to similar reasons. All intake variables were adjusted for total energy by taking the residual from a linear regression model in which total energy intake was the independent variable and the respective intake variable was the dependent variable ${ }^{(14)}$. We evaluated the association between general characteristics and either BLL or 8-OHdG by one-way ANOVA or simple regression. The Scheffé multiple comparison test was performed as appropriate ${ }^{(16)}$. Partial correlation analyses were used to find the relationship of dietary antioxidants with BLL or 8-OHdG.

We analysed the association between oxidative stress and intake variables in terms of BLL levels using multivariate linear regression models. We obtained the relative change of oxidative stress level by the inter-quartile range of the nutrients and the daily intake frequencies of fruit and vegetables in Q1-Q3 BLL groups as compared to the Q4 BLL group. The analytical models included main and interactive effects of BLL and the intake variable as well as key covariates including age, education, BMI, total energy intake as continuous variables and sex, nutrition supplement, alcohol consumption, smoking and exercise as categorical variables. There was no difference of interactive effects between BLL and dietary variables by sex; and thus we reported data for the total sample. The results were reported as $\log 10 \beta$-coefficients with their standard errors of oxidative stress (8-OHdG). Significance was set at $P<0 \cdot 05$.

\section{Results}

The participants' BLL, except for a few cases, were relatively normally distributed (Fig. 1). The geometric mean of BLL was close to $4 \mu \mathrm{g} / \mathrm{dl}$ (Table 1 ). The geometric mean of the highest quartile BLL group $(6.78 \mu \mathrm{g} / \mathrm{dl})$ was 2.9-fold higher than the lowest quartile group of BLL $(2 \cdot 36 \mu \mathrm{g} / \mathrm{dl})$. Cut-off points of BLL were $\leq 3.18$ and $>5.36 \mu \mathrm{g} / \mathrm{dl}$ for the lowest and the highest BLL groups, respectively. Only $1.0 \%$ had a BLL $>10 \mu \mathrm{g} / \mathrm{dl}$, which is a critical value established by the

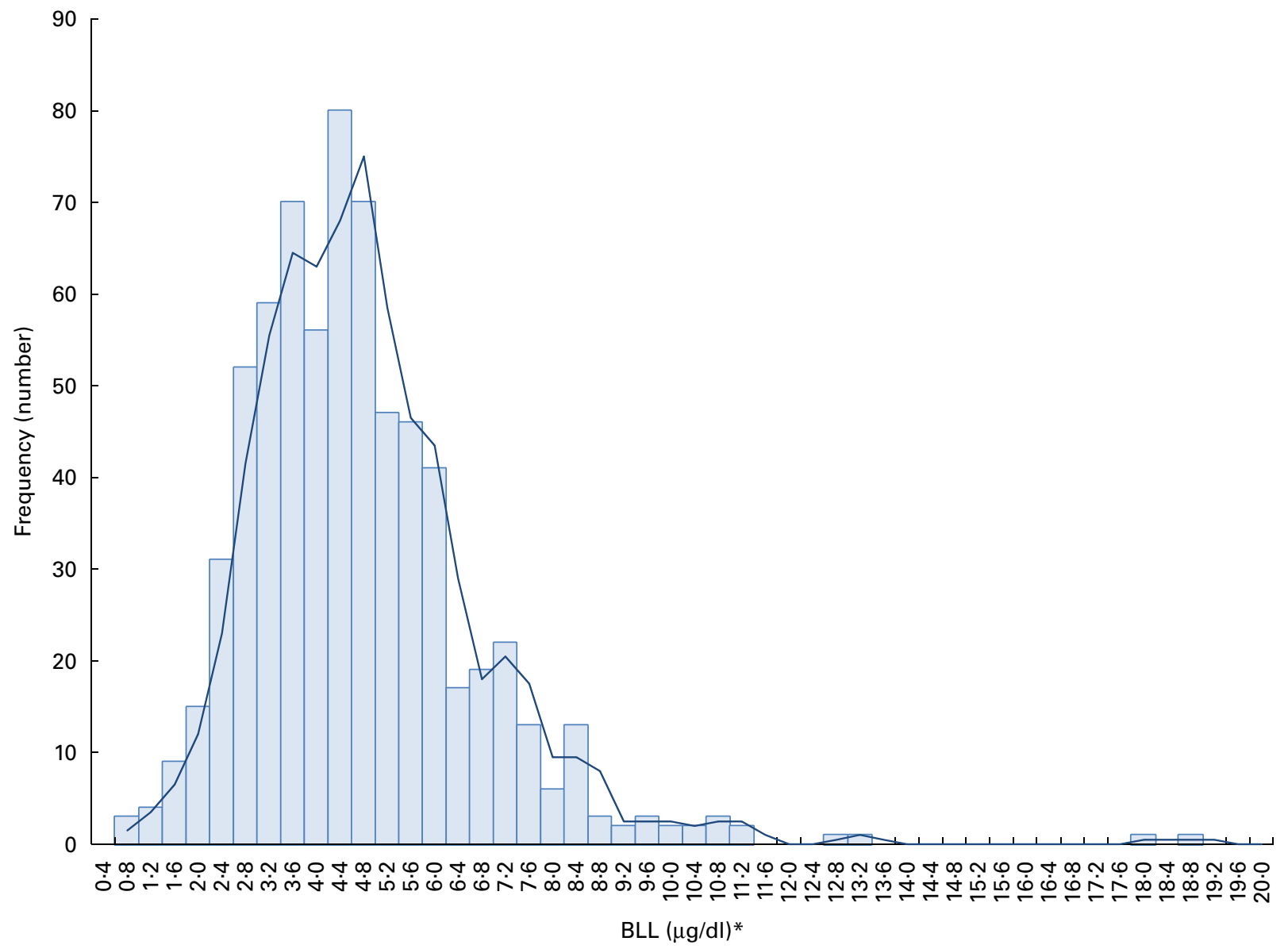

Fig. 1. Distribution of blood lead levels (BLL) by frequency $\left(n\right.$ 683). ${ }^{*} \mathrm{SI}$ unit conversion: BLL (mmol/l) $=0.04826 \times$ BLL ( $\left.\mu \mathrm{g} / \mathrm{dl}\right)$. (A colour version of this figure can be found online at http://www.journals.cambridge.org/bjn) 
Table 1. Blood lead levels (BLL) and urinary 8-hydroxy-2'-deoxyguanosine (8-OHdG) levels by BLL groups

(Mean values and standard deviations, $n$ 683)

\begin{tabular}{|c|c|c|c|c|c|c|c|c|c|c|}
\hline \multirow[b]{2}{*}{ BLL } & \multicolumn{5}{|c|}{$\mathrm{BLL}^{*}(\mu \mathrm{g} / \mathrm{dl})$} & \multicolumn{5}{|c|}{ 8-OHdG† ( $\mu \mathrm{g} / \mathrm{g}$ creatinine) } \\
\hline & Mean & SD & GM & GSD & Range & Mean & SD & GM & GSD & Range \\
\hline Q1 & 2.5 & 0.6 & 2.4 & 1.4 & $0.5-3.2$ & $2 \cdot 6$ & 0.8 & 2.4 & 1.5 & $0.3-3 \cdot 8$ \\
\hline Q2 & 3.7 & 0.3 & 3.7 & $1 \cdot 1$ & $3 \cdot 2-4 \cdot 2$ & $4 \cdot 7$ & 0.5 & $4 \cdot 7$ & 1.5 & $3.9-5.6$ \\
\hline Q3 & $4 \cdot 7$ & 0.3 & 4.7 & $1 \cdot 1$ & $4 \cdot 2-5 \cdot 4$ & $6 \cdot 7$ & 0.7 & $6 \cdot 6$ & 1.5 & $5 \cdot 6-8 \cdot 0$ \\
\hline Q4 & $7 \cdot 0$ & 1.9 & $6 \cdot 8$ & $1 \cdot 2$ & $5 \cdot 4-18 \cdot 6$ & $12 \cdot 3$ & $6 \cdot 2$ & 11.5 & 1.6 & $8 \cdot 0-60.7$ \\
\hline Total & 4.5 & 1.9 & $4 \cdot 1$ & 1.5 & & $6 \cdot 6$ & $4 \cdot 8$ & $5 \cdot 4$ & 1.5 & \\
\hline
\end{tabular}

GM, geometric mean; GSD, geometric standard deviation; $Q$, quartile.

${ }^{*}$ SI unit conversion: $\mathrm{BLL}(\mathrm{mmol} / \mathrm{l})=0.04826 \times \mathrm{BLL}(\mu \mathrm{g} / \mathrm{dl})$.

†SI unit conversion: $8-\mathrm{OHdG}(\mu \mathrm{mol} / \mathrm{mol})=0.399 \times 8-\mathrm{OHdG}(\mu \mathrm{g} / \mathrm{g}$ creatinine $)$.

US Centers for Disease Control and Prevention (CDC) ${ }^{(17)}$. The geometric mean of urinary $8-\mathrm{OHdG}$ was $5.43 \mu \mathrm{g} / \mathrm{g}$ creatinine, showing a wide range of distribution. The level of urinary $8-\mathrm{OHdG}$ was $4 \cdot 7$-fold greater in highest quartile group of $8-\mathrm{OHdG}$ than the lowest 8 -OhdG group.

The mean age of the participants was 51.4 years and approximately half of them were females (Table 2). Proportions of current smokers and drinkers were 21 and 55\%, respectively. BLL were higher in participants that were males, smokers, alcohol drinkers or supplement non-users (Table 2). Excretion of urinary 8-OHdG was positively related to age, alcohol consumption and supplement use. (Number of studies, percentages, mean values and standard deviations, $n$ 683)
Among the participants, 50 and $65 \%$ met the Korean Dietary Reference Intakes (DRI) ${ }^{(18)}$ for vitamin A and C, respectively (Table 3). Approximately one-third of them had vitamin $\mathrm{E}$ and energy intakes above the Korean DRI. Participants consumed 3.3 and 3.5 servings of kimchi and other vegetables, respectively, as well as 1.0 serving of fruits per day, on average. Intakes of antioxidant nutrients and related foods were not associated with BLL and 8-OHdG after controlling for age and sex.

In multivariate analyses, there were significantly greater negative associations of $8-\mathrm{OHdG}$ with vitamin $\mathrm{C}$ and $\mathrm{E}$ intakes in the lowest quartile group of BLL $(\leq 3 \cdot 18 \mu \mathrm{g} / \mathrm{dl})$ as compared

Table 2. Background characteristics and their association with blood lead levels (BLL) and urinary 8-hydroxy-2'-deoxyguanosine (8-OHdG) levels

\begin{tabular}{|c|c|c|c|c|c|c|c|c|}
\hline & \multirow[b]{2}{*}{$n$} & \multirow[b]{2}{*}{$\%$} & \multicolumn{3}{|c|}{$\mathrm{BLL}^{*}(\mu \mathrm{g} / \mathrm{dl})$} & \multicolumn{3}{|c|}{$8-\mathrm{OHdG} \dagger(\mu \mathrm{g} / \mathrm{g}$ creatinine) } \\
\hline & & & Mean & SD & $P \neq$ & Mean & SD & $P \neq$ \\
\hline Age (years) & & & $-0.001 \S$ & $0.001 \|$ & 0.31 & $0.007 \S$ & $0.001 \|$ & $<0.01$ \\
\hline Mean & & & & & & & & \\
\hline SD & & & & & & & & \\
\hline $\operatorname{BMI}\left(\mathrm{kg} / \mathrm{m}^{2}\right)$ & & & $0.006 \S$ & $0.003 \|$ & 0.03 & $-0.006 \S$ & $0.004 \|$ & 0.12 \\
\hline Mean & & & & & & & & \\
\hline SD & & & & & & & & \\
\hline Sex & & & & & $<0.01$ & & & 0.07 \\
\hline Male & 359 & $52 \cdot 6$ & 4.7 & 1.4 & & $5 \cdot 2$ & 1.9 & \\
\hline Female & 324 & 47.4 & 3.5 & 1.6 & & $5 \cdot 7$ & 1.9 & \\
\hline Smoking status & & & & & $<0.01$ & & & 0.23 \\
\hline Never smoker & 360 & $56 \cdot 8$ & $3.7^{\mathrm{a}}$ & 1.6 & & $5 \cdot 6$ & 1.9 & \\
\hline Past smoker & 139 & 21.9 & $4 \cdot 6^{\mathrm{b}}$ & 1.4 & & $5 \cdot 1$ & 1.9 & \\
\hline Current smoker & 135 & 21.3 & $4.9^{b}$ & 1.4 & & $5 \cdot 2$ & 1.9 & \\
\hline Alcoholic beverage use & & & & & $<0.01$ & & & 0.04 \\
\hline Never drinker & 251 & 38.7 & $3.6^{\mathrm{a}}$ & 1.6 & & 5.7 & 1.9 & \\
\hline Past drinker & 41 & $6 \cdot 3$ & $4 \cdot 5^{\mathrm{b}}$ & 1.4 & & $6 \cdot 1$ & 1.9 & \\
\hline Current drinker & 357 & $55 \cdot 0$ & $4 \cdot 5^{\mathrm{b}}$ & 1.5 & & $5 \cdot 1$ & 1.9 & \\
\hline Current exercise & & & & & 0.70 & & & 0.93 \\
\hline No & 282 & $42 \cdot 3$ & $4 \cdot 1$ & 1.5 & & 5.5 & 1.9 & \\
\hline Yes & 384 & $57 \cdot 7$ & $4 \cdot 1$ & 1.6 & & 5.5 & 1.8 & \\
\hline Current supplement use & & & & & $<0.01$ & & & 0.03 \\
\hline No & 384 & 63.4 & 4.3 & 1.5 & & $5 \cdot 1$ & 1.9 & \\
\hline Yes & 222 & $36 \cdot 6$ & $3 \cdot 8$ & 1.6 & & $5 \cdot 7$ & 1.9 & \\
\hline Education level & & & & & $0 \cdot 19$ & & & 0.25 \\
\hline$<$ High school & 166 & $26 \cdot 7$ & 4.0 & 1.5 & & $5 \cdot 8$ & 1.9 & \\
\hline High school & 241 & 38.7 & $4 \cdot 2$ & 1.5 & & $5 \cdot 2$ & 1.9 & \\
\hline$>$ High school & 216 & 34.7 & 3.9 & 1.6 & & $5 \cdot 4$ & 1.9 & \\
\hline
\end{tabular}

a,b Mean values within a column with unlike superscript letters were significantly different $(P<0.05$, Scheffé multiple comparison test).

${ }^{*}$ SI unit conversion: BLL $(\mathrm{mmol} / \mathrm{l})=0.04826 \times \mathrm{BLL}(\mu \mathrm{g} / \mathrm{dl})$.

$\dagger$ SI unit conversion: $8-\mathrm{OHdG}(\mu \mathrm{mol} / \mathrm{mol})=0.399 \times 8-\mathrm{OHdG}(\mu \mathrm{g} / \mathrm{g}$ creatinine $)$

$\ddagger$ Simple regression or one-way ANOVA.

$\S \log 10 \beta$

|| Log10 SE. 
Table 3. Daily antioxidant-related nutrient and food intakes and their associations with blood lead levels (BLL) and urinary 8-hydroxy-2'-deoxyguanosine (8-OHdG) levels

(Mean values and standard deviations, medians and ranges, $n$ 683)

\begin{tabular}{|c|c|c|c|c|c|c|c|c|c|}
\hline \multirow[b]{2}{*}{ Diet } & \multirow[b]{2}{*}{ Mean } & \multirow[b]{2}{*}{ SD } & \multirow[b]{2}{*}{ Median } & \multirow[b]{2}{*}{ Range } & \multirow[b]{2}{*}{ Meeting KDRI (\%) } & \multicolumn{2}{|c|}{ BLL } & \multicolumn{2}{|c|}{ 8-OHdG } \\
\hline & & & & & & $r^{*}$ & $P$ & $r^{*}$ & $P$ \\
\hline \multicolumn{10}{|l|}{ Total energy } \\
\hline $\mathrm{kJ}$ & 7683 & 2554 & 7331 & $6012-8834$ & $32 \cdot 1$ & -0.066 & 0.085 & -0.010 & 0.789 \\
\hline kcal & 1835 & 610 & 1751 & $1436-2110$ & & & & & \\
\hline Vitamin A ( $\mu \mathrm{g} R E)$ & 564 & 409 & 461 & $290-719$ & $49 \cdot 3$ & 0.023 & 0.510 & 0.025 & 0.514 \\
\hline Vitamin C (mg) & 117 & 87 & 97 & $58-153$ & 64.7 & -0.003 & 0.933 & 0.033 & 0.391 \\
\hline$\beta$-Carotene $(\mu \mathrm{g})$ & 2870 & 2240 & 2264 & $1402-3688$ & & 0.038 & 0.317 & 0.024 & 0.530 \\
\hline Vitamin E (mg $\alpha$-TE) & $9 \cdot 0$ & $5 \cdot 1$ & $7 \cdot 7$ & $5 \cdot 5-11 \cdot 2$ & $32 \cdot 4$ & -0.014 & 0.723 & 0.042 & 0.269 \\
\hline Vegetablest & $6 \cdot 8$ & 4.9 & $5 \cdot 6$ & $3 \cdot 5-9 \cdot 1$ & & 0.058 & 0.132 & -0.003 & 0.928 \\
\hline Kimchi & $3 \cdot 3$ & $2 \cdot 5$ & $3 \cdot 0$ & $1 \cdot 3-4 \cdot 6$ & & 0.069 & 0.074 & 0.011 & 0.773 \\
\hline Other vegetables & 3.5 & 3.5 & $2 \cdot 6$ & $1.3-4.3$ & & 0.022 & 0.566 & -0.010 & 0.785 \\
\hline All fruit (serving)‡ & 1.0 & 1.3 & 0.6 & $0 \cdot 2-1 \cdot 4$ & & -0.043 & 0.258 & 0.055 & 0.153 \\
\hline
\end{tabular}

KDRI, Korean Dietary Reference Intake; RE, retinol equivalent; TE, tocopherol equivalent.

* Spearman correlation coefficients with adjustment of age, sex and total energy except for total energy that was analysed including age and sex as covariates.

† Average one serving is approximately $50 \mathrm{~g}$ for kimchi and $35 \mathrm{~g}$ for other vegetables.

$\ddagger$ Average one serving size is approximately $150 \mathrm{~g}$.

to the highest quartile BLL group $(>5.36 \mu \mathrm{g} / \mathrm{dl})$ after controlling for potential confounders (Table 4). The magnitude of the association was higher for vitamin E than vitamin C. Similarly, the relationship between the vegetable intake, except for kimchi, and the 8-OHdG concentration was greater in the lowest quartile group of BLL than the highest BLL group. The associations between $8-\mathrm{OHdG}$ and the intakes including vitamin A, $\beta$ carotene, kimchi and fruits did not differ by BLL. There was a main effect of BLL on 8 -OHdG when the model included vegetables or fruits as an intake variable.

\section{Discussion}

Our cross-sectional study on urban adults showed that BLL modified the relationship between dietary antioxidants and oxidative stress. We found more of a decrease of oxidative stress by the increase of dietary antioxidants only in those from the lowest quartile of BLL $(\leq 3 \cdot 18 \mu \mathrm{g} / \mathrm{dl})$ compared with their counterparts in the highest quartile of BLL $(>5.36 \mu \mathrm{g} / \mathrm{dl})$. These findings show that dietary antioxidant intakes play a role regarding the reduction of oxidative stress, particularly when accompanied with low BLL $(\leq 3.18 \mu \mathrm{g} / \mathrm{dl})$ in urban adults. Previous studies have also proposed a stronger effect of antioxidants in persons with low levels of lead exposure ${ }^{(19,20)}$; yet none of them have attempted to examine the interaction effect of BLL and dietary antioxidant in relation to oxidative stress like the present study.

Arora et $a l .{ }^{(12)}$ reported that maternal dietary intake of PUFA modified the transfer of lead from maternal bone to breast milk. Among 301 Mexican women, an increase in patella lead was associated with higher breast milk lead level in the lowest tertile of the PUFA group compared with the highest tertile of the PUFA group ${ }^{(12)}$. Previous studies have proposed a stronger effect of antioxidants on BLL in persons with low lead exposure ${ }^{(10,20)}$. Blood nutrient levels were reported to be related to hazardous substances in the high-exposure group $^{(21)}$. In pregnant Bangladeshi women, concentrations of metabolites of inorganic arsenic were inversely associated with plasma folate and $\mathrm{Zn}$ levels in only those with high arsenic exposure $(>209 \mu \mathrm{g} / \mathrm{l} \text { of urinary arsenic })^{(21)}$.

The modifying role of BLL found in the present study is relevant to the inverse role of BLL and dietary antioxidants regarding oxidative stress. $8-\mathrm{OHdG}$ is one of the most critical lesions generated from deoxyguanosine by oxygen free radicals; and thus urinary $8-\mathrm{OHdG}$ is considered a measure of DNA oxidation in response to free radicals ${ }^{(22,23)}$. Antioxidants are thought to be effective free-radical scavengers while protecting DNA from oxidative damage. Vitamin $\mathrm{C}$ has been suggested as a possible chelator of lead, with similar potency to that of EDTA $^{(24)}$. Vitamin E, known to protect biological membranes and lipoproteins from oxidative stress, was reported to prevent lead from affecting the production of free radicals in the liver ${ }^{(25)}$. $\beta$-Carotene was thought to mediate lipid peroxidation ${ }^{(1)}$.

Conversely, lead exposure has been shown to induce oxidative stress ${ }^{(1,5)}$. High to moderate doses of lead exposure appeared to generate free radicals, which in turn result in oxidative damage to critical biomolecules, lipids, proteins and $\mathrm{DNA}^{(1)}$. In epidemiological studies, adverse health impacts of BLL were also found not only in high-level but also low-level lead exposure populations. Among adults with low-lead exposure (mean BLL of $2 \cdot 8 \mu \mathrm{g} / \mathrm{dl}$ ), there was a graded positive association between BLL and serum $\gamma$-glutamyltransferase, a marker of oxidative stress ${ }^{(5)}$. BLL was associated with enhanced homocysteine levels, in adults with a mean BLL of $3.5 \mu \mathrm{g} / \mathrm{dl}^{(6)}$. Considering the links between antioxidant nutrient or related food intakes and oxidative damage $\mathrm{e}^{(19,26)}$, it is important that the role of nutrition on health outcomes is modified by the degree of lead levels even in populations with a low-level lead exposure.

We also found a greater inverse relationship between vegetables excluding kimchi and $8-\mathrm{OHdG}$ excretion in the lowest BLL group than the highest BLL group. Kimchi, a pickled and fermented cabbage or radish, is the most 
important source of vegetables in Korea. Kimchi is also a major intake source of $\mathrm{Na}$, which is a risk factor for hypertension and elevated blood pressure, as is lead exposure ${ }^{(1)}$. Animal studies have shown that high salt intake enhances blood pressure during the development of hypertension via oxidative stress ${ }^{(27,28)}$. For this reason, we divided vegetables into kimchi and other vegetables excluding kimchi. Only the intake of other vegetables showed a greater inverse association with the oxidative stress marker $8-\mathrm{OHdG}$ in participants with the lowest quartile of BLL $(\leq 3.18 \mu \mathrm{g} / \mathrm{dl})$ than those in the highest BLL group $(>5.36 \mu \mathrm{g} / \mathrm{dl})$. Thus, it is plausible to assume that the beneficial role of vegetable intake is weakened by its $\mathrm{Na}$ content in addition to the extent of lead exposure.

Unlike other vegetable intake, we found a null association between fruit intake and oxidative stress regardless of BLL in multivariate analyses. Similar to our findings, a review of cancer risk also reported that vegetable rather than fruit intake had a more beneficial role with respect to the reduction of cancer risk ${ }^{(29)}$. Besides, a uniformly low consumption of fruit in the participants of this study would be one of the reasons for the null relationship of fruit consumption with outcome measures. Median fruit intake ( 0.6 servings/d) of our participants was about $10 \%$ of vegetable consumption (5.6 servings/d).

Our study has some limitations. Even though we used a dichotomous measure of nutrition supplement intake, a lack of quantitative assessment of antioxidant nutrient supplements may underestimate true associations of antioxidants with BLL and oxidative stress. As with any other dietary studies using FFQ, recall bias would be a concern. We used the immunoassay methodology utilising primary monoclonal antibody (clone N45.1) to detect 8-OHdG. However, this ELISA assay could lead to overestimation of 8 -OHdG because a variety of components in urine, particularly urea, can cross-react with the N45.1 antibody ${ }^{(30)}$. Therefore, urea removal by enzymatic decomposition by urease would have improved accuracy of the measurements, but we did not treat urease before measurement of 8-OHdG. Finally, the cross-sectional nature of this study may preclude generalising the results of the study to urban adults as a whole.

This is the first study to assess the modifying role of BLL in the association between dietary antioxidants and oxidative stress. Moreover, our study is the largest study available to measure oxidative stress, BLL and dietary intake in the general population in Korea to the best of our knowledge.

In conclusion, the finding of this study that dietary antioxidants more significantly reduced oxidative stress in the lowBLL $(\leq 3.18 \mu \mathrm{g} / \mathrm{dl})$ group than the high-BLL $(>5.36 \mu \mathrm{g} / \mathrm{dl})$ group supports a rationale for increasing the intake of dietary antioxidants besides lowering BLL in the urban population.

\section{Acknowledgements}

The authors declare no conflicts of interest. Y.-C. H., J.-H. L. and E.-H. H. designed the research and conducted the research; S.-Y. O., S.-O. K., M.-S. P., and H. K. analysed the data; Y.-C. H. and S.-Y. O. wrote the paper. S.-Y. O. had 
primary responsibility for the final content. All authors read and approved the final version of the manuscript. This study was supported in part by a grant from Kyung Hee University, 2007.

\section{References}

1. Ahamed M \& Siddiqui MK (2007) Environmental lead toxicity and nutritional factors. Clin Nutr 26, 400-408.

2. Courtois E, Marques M, Barrientos A, et al. (2003) A. Leadinduced downregulation of soluble guanylate cyclase in isolated rat aortic segments mediated by reactive oxygen species and cyclooxygenase-2. J Am Soc Nephrol 14, 1464-1470.

3. Ahamed M, Verma S, Mumar A, et al. (2006) Delta-aminolevulinic acid dehydratase inhibition and oxidative stress in relation to blood lead among urban adolescents. Hum Exp Toxicol 25, 547-553.

4. Dursun N, Dogan P \& Donmez H (2001) Plasma and erythrocyte lipid peroxide levels in workers with occupational exposure to lead. Biol Trace Elem Res 82, 29-34.

5. Lee DH, Lim JS, Song K, et al. (2006) Graded associations of blood lead and urinary cadmium concentrations with oxidative-stress-related markers in the U.S population: results from the third National Health and Nutrition Examination Survey. Environ Health Perspect 114, 350-354.

6. Schafer JH, Glass TA, Bressler J, et al. (2005) Blood lead is a predictor of homocysteine levels in a population-based study of older adults. Environ Health Perspect 113, 31-35.

7. Menke A, Muntner P, Batuman V, et al. (2006) Blood lead below 0.48 micromol/l $(10$ microg/dL $)$ and mortality among US adults. Circulation 114, 1388-1394.

8. Mayne ST (2003) Antioxidant nutrients and chronic disease: use of biomarkers of exposure and oxidative stress status in epidemiologic research. J Nutr 133, 933S-940S.

9. Young IS \& Woodside JV (2001) Antioxidants in health and disease. J Clin Pathol 54, 176-186.

10. Loft S, Fischer-Nielsen A, Jeding IB, et al. (1993) 8-Hydroxydeoxyguanosine as a urinary biomarker of oxidative DNA damage. J Toxicol Environ Health 40, 391-404.

11. Dawson EB, Evans DR, Harris WA, et al. (1999) The effect of ascorbic acid supplementation on the blood lead levels of smokers. J Am Coll Nutr 18, 166-170.

12. Arora M, Ettinger AS, Peterson KE, et al. (2008) Maternal dietary intake of polyunsaturated fatty acids modifies the relationship between lead levels in bone and breast milk. J Nutr 138, 73-79.

13. Vahter ME (2007) Interactions between arsenic-induced toxicity and nutrition in early life. J Nutr 137, 2798-2804.

14. Willett W \& Stampfer MJ (1986) Total energy intake: implications for epidemiologic analyses. Am J Epidemiol 124, $17-27$.
15. Ahn Y, Kwon E, Shim JE, et al. (2007) Validation and reproducibility of food frequency questionnaire for Korean genome epidemiologic study. Eur J Clin Nutr 61, $1435-1441$.

16. Cody RP \& Smith JK (2005) Applied Statistics and SAS Programming Language, 5th ed. New York, NY: Prentice-Hall.

17. CDC (2005) Preventing Lead Poisoning in Young Children, a Statement by the Centers for Disease Control and Prevention. http://www.cdc.gov/nceh/lead/publications/prevleadpoisoning.pdf (accessed 1 May 2010).

18. Korean Nutrition Society (2005) Dietary Reference Intakes for Koreans. Seoul: Guk Jin Gi Hwak.

19. Loft S, Møller P, Cooke MS, et al. (2008) Antioxidant vitamins and cancer risk: is oxidative damage to DNA a relevant biomarker? Eur J Nutr 47, 19S-28S

20. Hsu P-C \& Guo YL (2002) Antioxidant nutrients and lead toxicity. Toxicology 180, 33-44.

21. Li L, Ekström EC, Goessler W, et al. (2008) Nutritional status has marginal influence on the metabolism of inorganic arsenic in pregnant Bangladeshi women. Environ Health Perspect 116, 315-321.

22. Arimoto T, Yoshikawa T, Takano H, et al. (1999) Generation of reactive oxygen species and 8-hydroxy-2'-deoxyguanosine formation from diesel exhaust particle components in L1210 cells. Jpn J Pharmacol 80, 49-54.

23. Harri M, Svoboda P, Mori T, et al. (2005) Analysis of 8-hydroxydeoxyguanosine among workers exposed to diesel particulate exhaust: comparison with urinary metabolites and PAH air monitoring. Free Radic Res 39, 963-972.

24. Goyer RA \& Cherion MG (1979) Ascorbic acid and EDTA treatment of lead toxicity in rats. Life Sci 24, 433-438.

25. Packer L (1991) Protective role of vitamin E in biological systems. Am J Clin Nutr 53, 1050S-1055S.

26. Li N, Jia X, Chen CY, et al. (2007) Almond consumption reduces oxidative DNA damage and lipid peroxidation in male smokers. J Nutr 137, 2717-2722.

27. Koga Y, Hirooka Y, Araki S, et al. (2008) High salt intake enhances blood pressure increase during development of hypertension via oxidative stress in rostral ventrolateral medulla of spontaneously hypertensive rats. Hypertens Res 31, 2075-2083.

28. Ketonen J \& Mervaala E (2008) Effects of dietary sodium on reactive oxygen species formation and endothelial dysfunction in low-density lipoprotein receptor-deficient mice on high-fat diet. Heart Vessels 23, 4204-4229.

29. Kim YI (2002) Nutrition and cancer. In Present Knowledge in Nutrition, 8th ed., pp. 573-589 [BA Bowman and RM Russell, editors]. Washington, DC: ILSI Press.

30. Song MF, Li YS, Ootsuyama Y, et al. (2009) Urea, the most abundant component in urine, cross-reacts with a commercial 8-OH-dG ELISA kit and contributes to overestimation of urinary 8-OH-dG. Free Radic Biol Med 47, 41-46. 